\title{
Hypoglycemic activity study of the novel bornyl derivatives of p-(benzyloxy)phenylpropionic acid
}

Darya Kuznetsova, Mikhail Khvostov*, Sergey Kuranov, Olga Luzina, Dmitriy Baev, Nataliya Zhukova, Tatyana Tolstikova and Nariman Salakhutdinov

\section{Introduction}

Type 2 diabetes mellitus (T2DM) is a serious medical and social problem because the prevalence of this disease has been increasing at an alarming rate. The several classes of antihyperglycemic drugs are used to treat T2DM, but each has its own adverse effects. The main adverse effects of modern oral antidiabetic agents include hypoglycemia, fluid retention, osteoporosis, and heart failure, which limit their clinical use. Therefore, the development of new antidiabetic candidate compounds for the control of various parameters of metabolic disturbances with minimization of adverse effects remains the primary and most important task in the treatment of T2DM. In this study we explore two bornyl derivatives of $\mathrm{p}$ (benzyloxy)phenylpropionic acid 1 and 2 for their ability to normalize glucose metabolism in mice with metabolic disorders.

\section{Results}

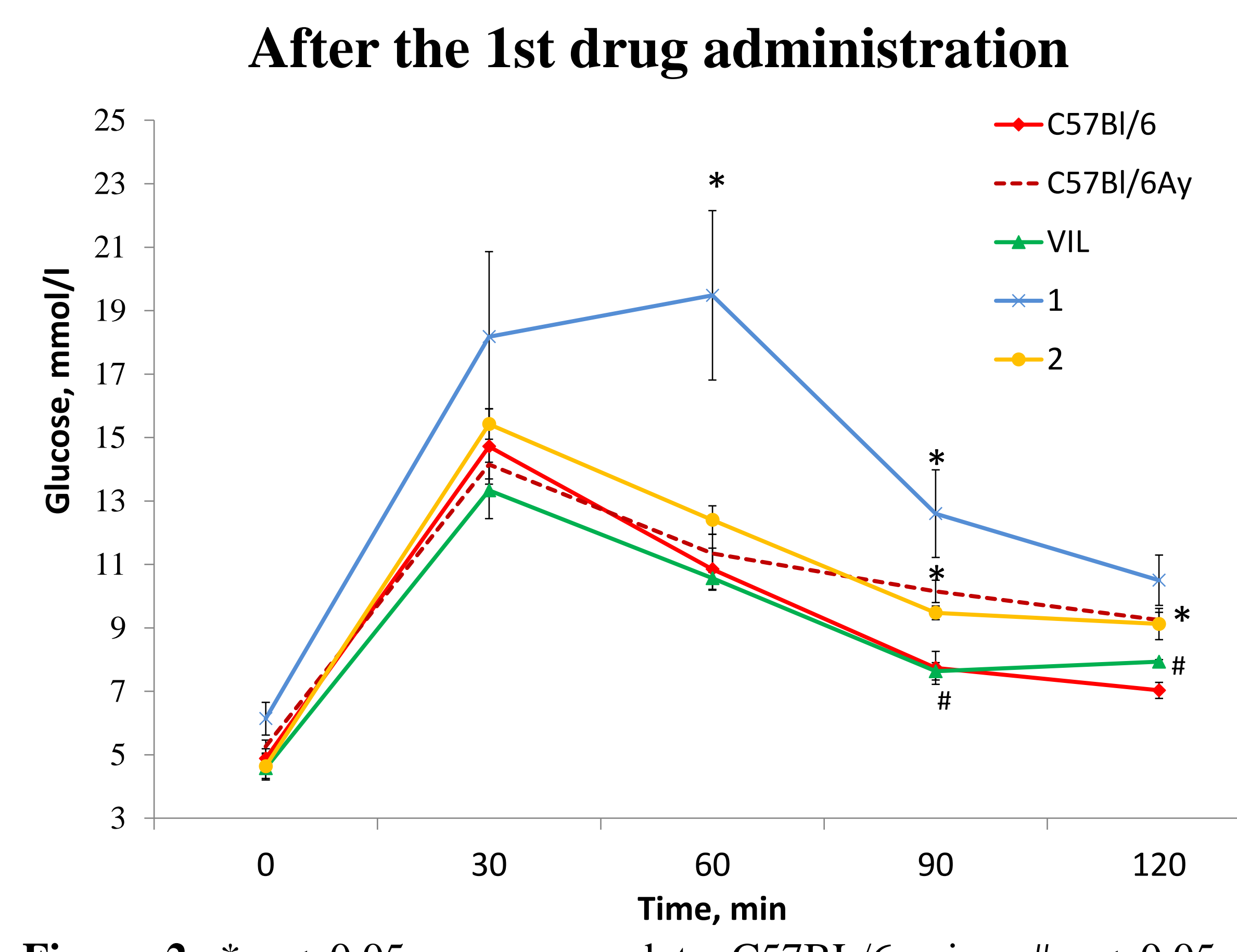

Figure 2. ${ }^{*} \mathrm{p}<0.05$ as compared to C57BL/6 mice, $\# \mathrm{p}<0.05$ as compared to untreated AY mice. OGTT immediately performed after the first oral introduction of studied compounds to AY mice did not show ahypoglycemic effect but revealed the impaired glucose tolerance in untreated mice. Conversely administration of 1 exerted a hyperglycemic effect.

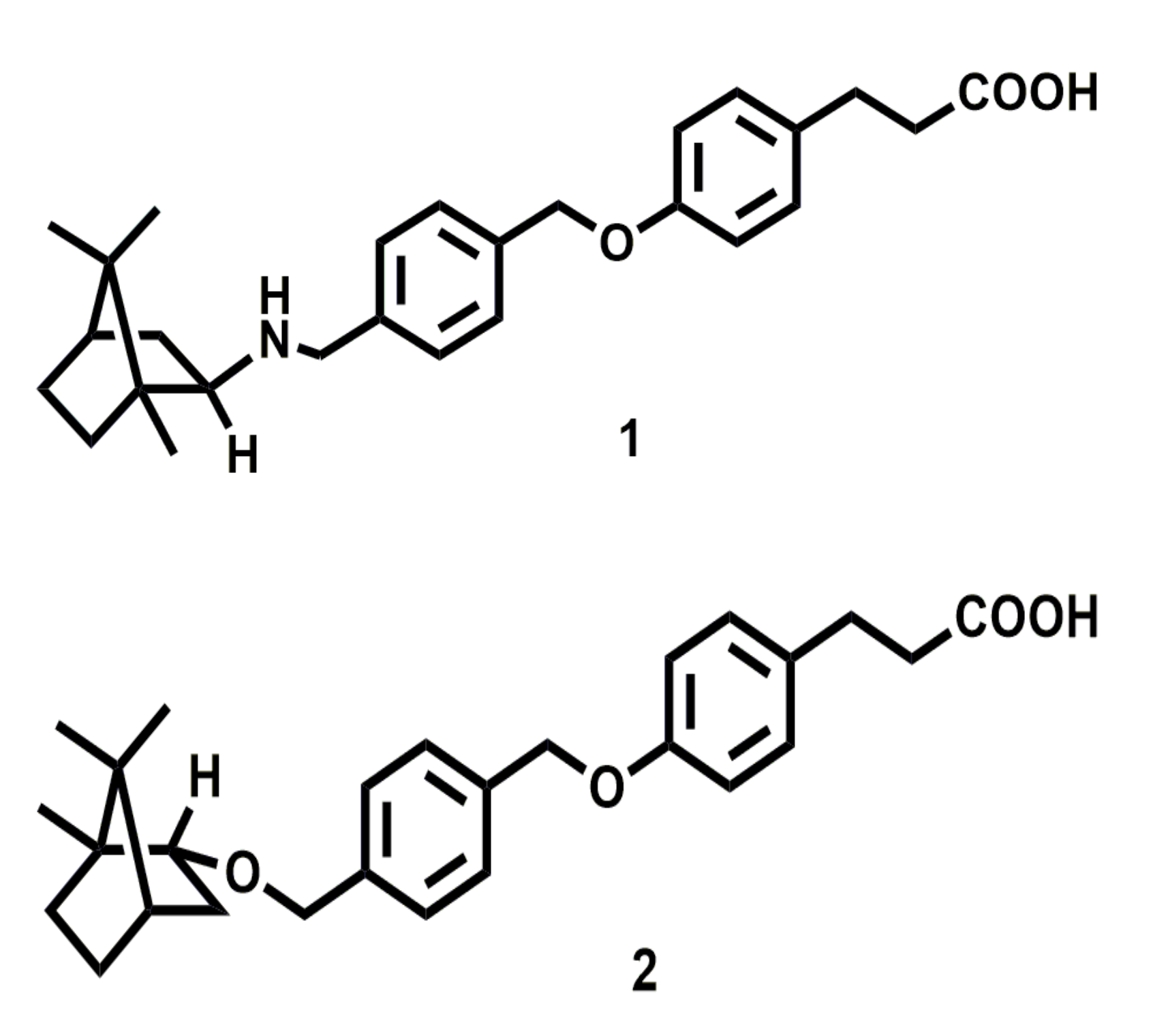

Figure 1. New bornyl derivatives.

The OGTT in AY mice

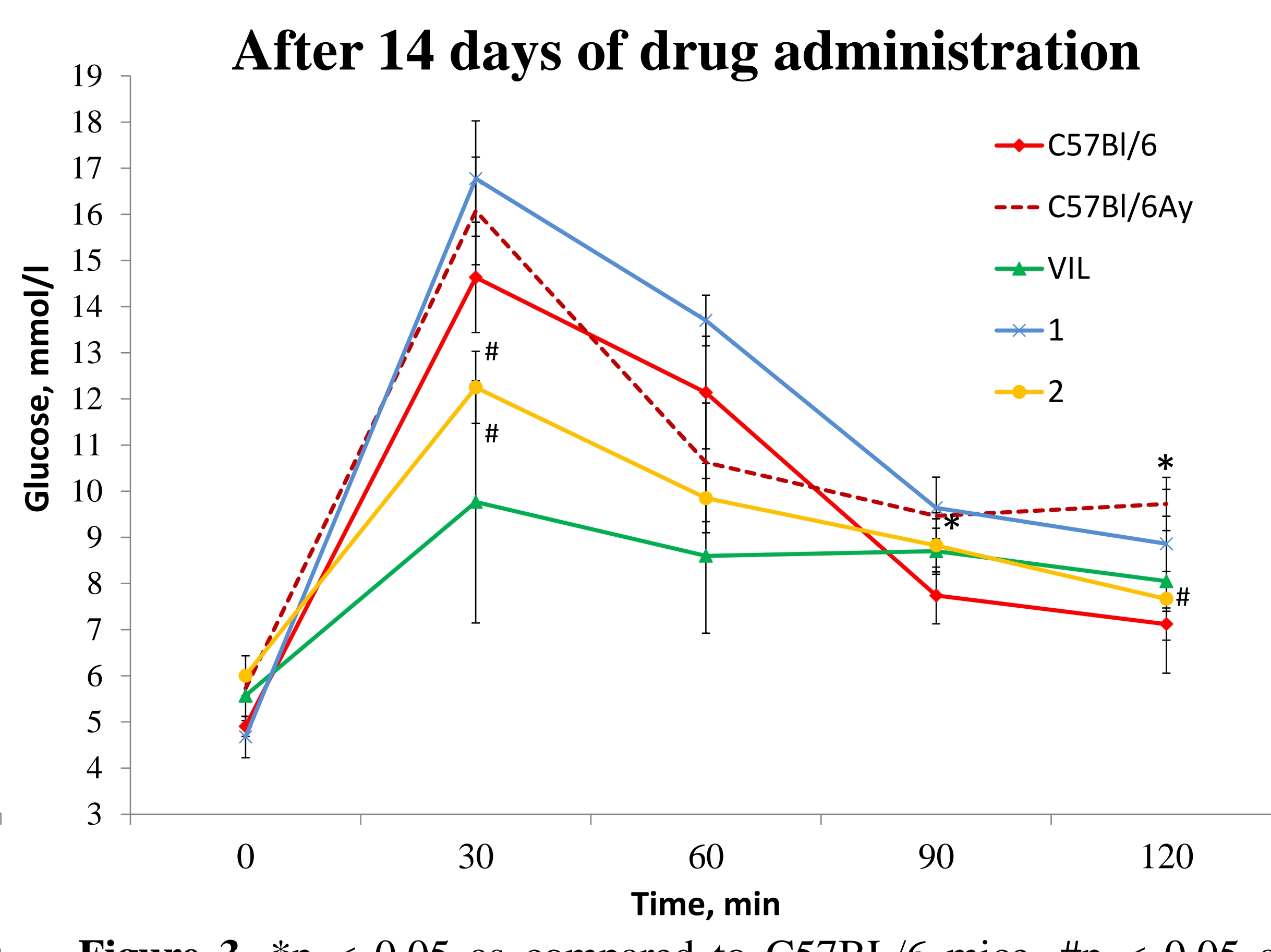

Figure 3. ${ }^{*} p<0.05$ as compared to C57BL/6 mice, \#p $<0.05$ as compared to untreated AY mice. After 14 days of administration, another OGTT was carried out, where it was found that glucose tolerance in untreated AY mice remained abnormal. At the same time, a significant hypoglycemic effect was detected in mice treated by 2 .

\section{Histological examination}

Histological examination of the liver of a $\mathrm{C} 57 \mathrm{BL} / 6$ mouse (A) and an untreated AY mouse (B-D) at the end of the experiment
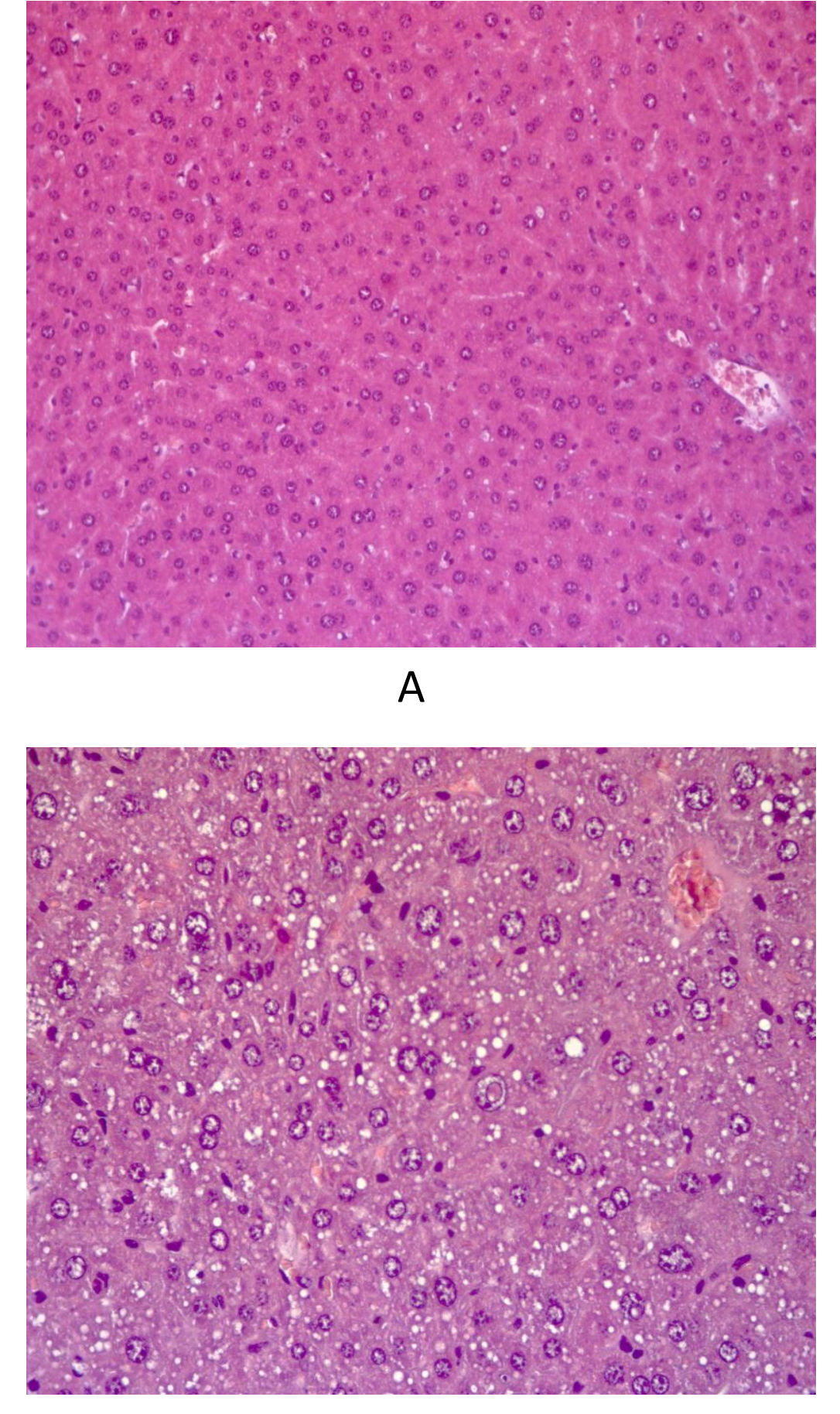

Figure 5. A. Nuclear polymorphism of hepatocytes. Staining with hematoxylin and eosin, magnification $\times 200$. B Polymorphic lipid infiltration of hepatocytes. Staining with hematoxylin and eosinz magnification $\times 200$. C. Polymothic lipid infiltation, nuclear polymorphism. $\mathrm{C}$. Polym Staining with hem Staining with lipid infitation, no gycog. Staing: pen. D Polymorphic lipid infiltration, no glycogen. Staining: periodic acid-Schiff, hematoxylin, and orange G; magnification $\times 200$.
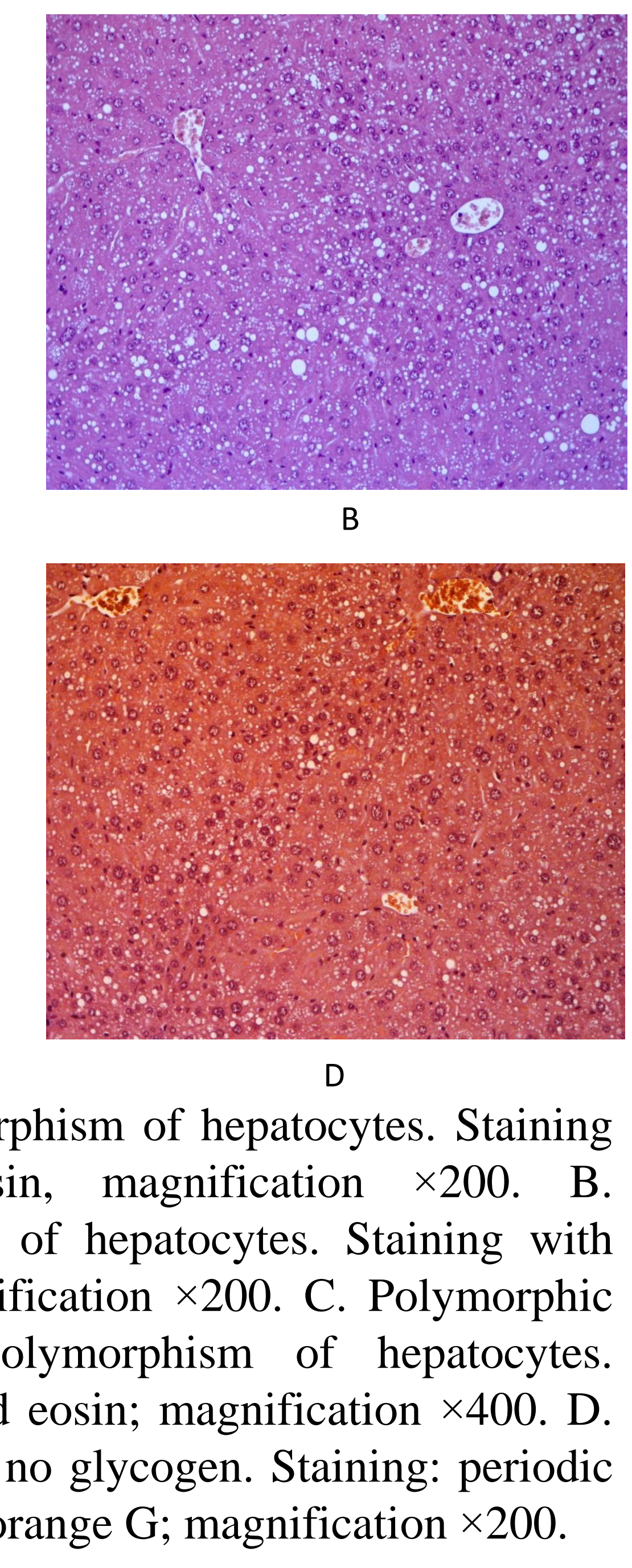

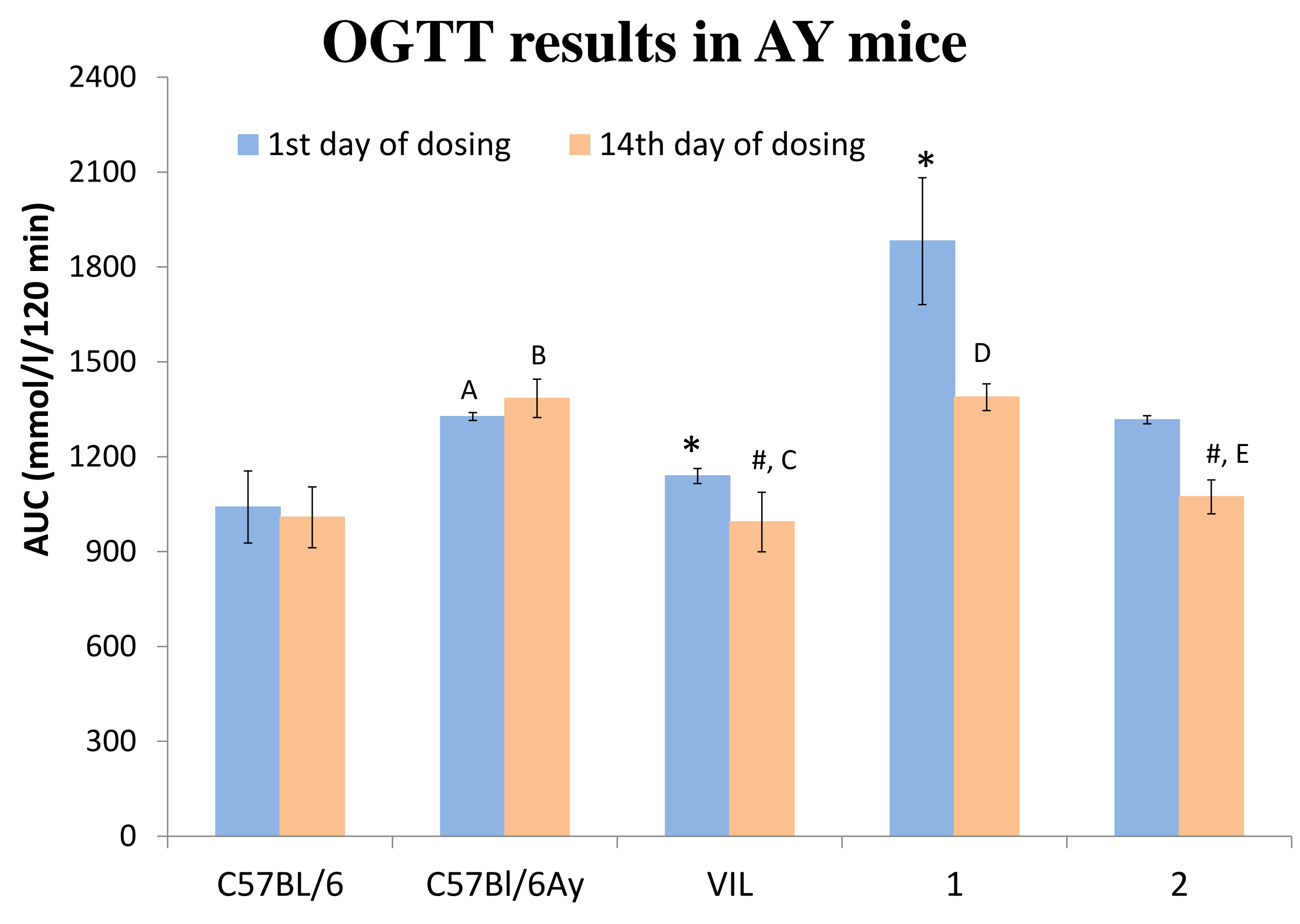

Figure 4. $* p<0.05$ vs. untreated AY mice on the 1st day of dosing; \#p 0.05 vs. untreated AY mice on the 14th day of dosing; Ap $<0.05$ vs. C57BL/6 mice on the 1st day of dosing; $\mathrm{Bp}<0.05$ vs. C57BL/6 mice on the 14th day of dosing; $\mathrm{Cp}<0.05$ vs. VIL 1st day of dosing; $\mathrm{Dp}<0.05$ vs. compound 1 on the 1 st day of dosing; Ep < 0.05 vs. 3 on the 1st day of dosing A significant hypoglycemic effect was detected in mice treated by 2 judging by a lower AUC. The hyperglycemic effect of 1 decreased by 2 judging by a lower $A U C$. The fle untreated AY group. The administration of VIL for 2 weeks also improved the results of the OGTT.
Histological examination of the liver of AY mice treated with VIL or compounds 1 or 2
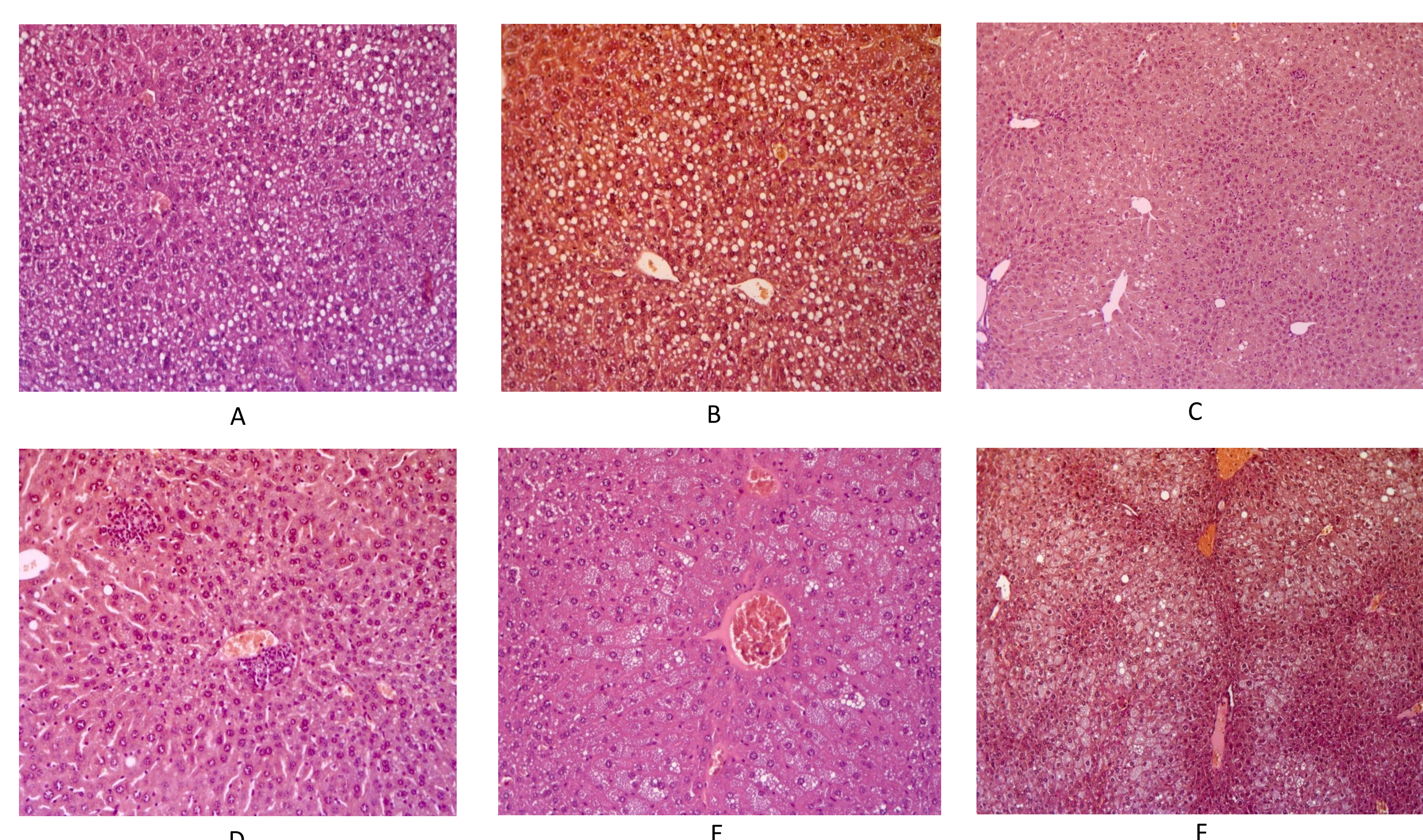

During histological examination of the mice's liver at the end of the experiment it was found that in animals received 1 for 14 days, there was alleviation of metabolic disturbances whereas is those who received 2 partial correction of the metabolic abnormalities was detected. In VIL group no correction of metabolic abnormalities was found. These differences suggest different mechanism of action of VIL and studied compounds. and hepatocyte polymorphism. Staining: hematoxylin and eosin; magnification $\times 200$. B. The liver of an AY mouse after 14 days of administration of VIL. Polymorphic lipid infiltration, no glycogen. Staining periodic acid-Schiff, hematoxylin, and orange $\mathrm{G}$ magnification $\times 200$. C. The liver of an AY mouse that received 1 for 14 days. Polymorphic lipid infiltration, no glycogen. Staining: periodic acid-Schiff, The liver of an AY mouse that received 1 for 14 days. Fine necrosis of hepatocytes. Staining: hematoxylin and eosin; magnification $\times 200$. E. The liver of an AY mouse who received 2 for 14 days. Small-vesicle fat dystrophy, hepatocyte hypertrophy. Staining with hematoxylin and eosin; magnification $\times 200$. F. The liver of an AY mouse that received 2 for 14 days. Small-vesicle fat dystrophy, no glycogen. Staining. periodic acid-Schiff, hematoxylin, orange G. magnification $\times 200$.

\section{Conclusion}

These results indicate that 1 and 2 have a good potential as new agents for diabetes mellitus treatment with different from VIL mechanism of action. Supported by RSF grant 20-13-00029. 